\title{
Analyzing the Negative Effects of Motivating e- Learning Tools in Archeology Teaching
}

\author{
M. A. Molinero-Polo, C. Hernández, D. M. Méndez- \\ Rodríguez \\ Universidad de La Laguna \\ Calle Molinos de Agua, s/n, 38207 San Cristóbal de La \\ Laguna, Santa Cruz de Tenerife, Spain
}

\begin{abstract}
In this article we study the negative effects of applying motivating e-Learning tools as a method to increase students' engagement through their learning process. In particular, we demonstrate that increasing students' motivation can have a negative effect on students' efficiency if they engage with the applications in a wrong way. In our carried out experience, we have used a virtual reconstruction of the TT 209 archeological site in Luxor. This application allows students to move inside and outside the site and get some information on the different activities that were done along the field work. We have found that students tend to use the application just as a game. This fact decreases students' efficiency since they do not pay enough attention to the learning activities inside the system. To avoid this effect, we propose to use gamification strategies such as rewards to redirect students' attention to the learning process.
\end{abstract}

Keywords-Virtual Reality, Egyptology, Motivation, Learning Tools, Engagement

\section{INTRODUCTION}

The use of computers as an alternative to traditional teaching techniques is much extended nowadays. Institutions are making great efforts applying e-Learning strategies to improve the quality and visibility of their courses $[1,2]$. Among the advantages found in the use of computers in education, one of the most relevant is the increasing of students' motivation. Students perceive the use of computers as a much more pleasant activity than just attending lectures. Many techniques and procedures have been developed recently in order to increase students' motivation by using computers. One of the most popular techniques is the use of gamification mechanisms, supported by the idea that computer-games are fun and introducing game-like features into e-Learning activities makes them more attractive $[3,4]$.

The use of gamification as a potentially motivating elearning tool is a hot topic nowadays and several studies [EDUCON] have focused, for example, on the dependence of the gamification benefits with the learner profile $[5,6]$. The influence of the learner's age has been also found as a factor that may have a strong influence in the positive influence of gamification, being the effect stronger in middle school participants than in adults $[7,8]$. Finally, another study found a clear difference between effectiveness and engagement in the use of gamification techniques, where effectiveness decreased as engagement increased [9]. It is clear that using computers in learning is a powerful technique that can have a huge impact in

\author{
S. Pérez-Ruiz, A. Acebo, F. Jurado, P. Rodríguez, S. \\ Atrio, G. M. Sacha \\ Universidad Autónoma de Madrid \\ Campus de Cantoblanco, 28049 Madrid, Spain
}

teaching and, therefore, must be treated carefully to avoid any potential negative effect.

The intrinsic nature of the different subjects under study is another key factor to motivate the development of e-Learning tools. There are some disciplines that can be easily presented in the classroom from an experimental point of view. It is easy to see a scientist showing some chemical reactions to a group of students, or a work group when students represent a Lope de Vega's comedy. However, those practices are much more difficult in other disciplines such as Archeology. The logistical problem of organizing trips for students to a real archeological site makes this activity a very restricted one, which can be carried out only in very specific time windows due to the inherited restrictions of the archaeological activities. This fact is even worse when we focus on Egyptology. This branch of archaeological studies and its specific techniques and characteristics are mainly developed in Egypt itself, where the logistical problems of possible visits highly increase. Fortunately, the extremely fast development of new technologies opens a new scenario where virtual worlds and multimedia resources can be used as a potential alternative to real visits to archaeological sites where access can be restricted by any reason.

In previous works we presented a virtual reconstruction of an archaeological site in Egypt (TT 209, Figure 1) [10], to recreate in a very immersive way the specific archaeological techniques used in Egyptology.



Fig. 1. TT 209 picture taken during the fourth season of field work on July 2015 
In that work we made use of virtual visits as a tool to improve students' motivation and attention. That tool was used as a virtual substitution of the real experience of being present at the site during the excavation process, which is not always possible in this discipline. The tool has been developed for young students and it is adequate for anyone who can read and use computers correctly. If it is not the case, the application can still be used as additional teaching material, being used by the teacher directly. In this case, questions and videos are shown to the whole class instead of individually [10].

In the present article we will explore the positive and negative effects of using e-Learning tools in Archeology. We will demonstrate that a lower performance can be found when using motivating tools. Additionally, we will show that using gamification techniques can be useful to avoid this negative effect.

\section{Virtual ReCONStruction OF THE TT209 Site}

The experiments related to the virtual reconstruction of the TT209 site involved three stages, as shown in Figure 2 [10]: Data acquisition; virtual visit tool implementation and pilot experience. These three stages are described in detail later.

TT 209 is located in Luxor, in its western necropolis, a worldwide known historical site. The Archaeological Mission of the University of La Laguna has developed four seasons of field work that have excavated the courtyard, the first of the underground chambers, and a mudbrick building of large dimensions on the surface. Through the inscriptions found, it has been possible to identify the name (Nisemro or Ashemro), the titles and the ethnic group of the tomb's proprietor. They have also allowed deducing the chronology of the monument, built at the beginning of the Twenty-fifth Dynasty, a period when Egypt was ruled by a royal family of Nubian origin. The interest of the site is that it is later to the best known phase of the Theban necropolis, the New Kingdom, and, therefore, informs of the cultural transformations in the post-empire Egypt. The architectural and decorative innovations can be seen as tests and adaptations of existing models to resolve the needs of Nubian elites to display prestige goods through a constructive and ritual policy. These novelties are the basis for the later development of Egyptian funerary architecture of the Late Period.

The first stage in the development of the virtual reconstruction of the TT 209 site was the data acquisition in the site. This task was performed by a team of computer scientist during the 2015 campaign, which extended from June to August. The subject under study was the Archaeological methodology instead of the history of the site itself. For this reason, the multimedia material obtained in this stage focused on the team instead of the tomb. The multimedia material needed for the reconstruction is related to the tomb dimensions and materials. Pictures and measures of both the interior and exterior of the site were taken, as well as accurate measurements of the dimensions already taken by the Archaeologists in previous campaigns. Several videos and pictures were additionally taken to include other complementary information in the application, such as questions about the tools used for different members of the expedition or the specific relevant activities that were developed.
The selected engine for the virtual visit was Unity 5.2.2. The $3 \mathrm{D}$ object set, including the whole volume of both the interior and exterior of the tomb, was developed by Blender 2.69. Some images from the final version of the application are shown in figure 2. Since this application has been described before [10], only a brief description is given here.

Two ways of interaction with the application are possible. In the first one, students can watch a set of videos about the different activities that can be done at the site when arriving to certain places. For example, in the video related to the photographer, he is preparing a photogrammetry system and, at the same time, explaining some details about his work in the audio. After the video ends, students can answer some questions about video contents. Second interactive activities are accessed through several red spheres that can be found in different places of the virtual world. These spheres are triggers of questions that are not directly related to the videos of the expedition members and are used to improve students' knowledge by reasoning.

Although the TT209 Virtual Visit can be used by students on diverse levels [10], only students with ability to read and use the application independently will be analyzed here.



Fig. 2: Stages followed to complete the experiment related to the virtual visit of TT209 in education organizations. Red arrows indicate the path followed from one stage to another. Grey arrows show some examples of the activities done in different stages

The strategy that we propose for students with good reading and computer skills starts with an oral presentation in which the main characteristics of the excavation and related jobs are shown.

In the initial talk only a brief explanation about the virtual visit is done, given that this kind of students will be using the application later autonomously. After the initial talk, a first exercise is proposed to the students (Practice 1 in Figure 3). This exercise is placed here as a control point and will measure the 
different students' performance before and after using the application. Then, students start using the application, being free to access to the learning material in the application, acquiring a better and wider sight of the Archeological excavation processes.



Fig. 3. Methodology proposed for students with correct reading skills and an efficient use of computers

A second exercise (practice 2), with a similar format than the previous one, is presented and developed by students when using the application. The format suggested here must be similar to Practice 1 in order to avoid experimental errors. We proposed the development of Practice 2 at this moment because students could be answering Practice 2 right after taking information from the application, and even watching videos again, for example, if needed. Last step in this methodology is an evalUAM test [11] where all the questions asked by the application are asked again to the students. This tests works as a control point that measures the knowledge acquired during the process. This test is done right after students finish using the application.

\section{PILOT EXPERIENCE}

The pilot experience was divided in two phases. The first one involved 4 education centers in Comunidad de Madrid. In IES1 and IES2, 101 and 106 students (around 12 years old) were included in the experience respectively. The two other organizations, CEIP1 and CEIP2, are public institutions in which we have worked with 44 and 40 students respectively from fifth and sixth courses of primary education (around 10 and 11 years old respectively).

In order to analyze students' answers, we divided their answer into three categories: a) empty answers or text that is not related to the activity at all, b) answers that are relatively correct and, finally, c) answers that, in general terms, are complete and correct. The former second category is the wider one, since many different answers with many different contents could be included there. Although that category could be divided into some subcategories, we decided to keep only one to reduce the influence of our subjective interpretation of the students' answers. Regarding the third category, it is worth noting that our open questions do not have a single and concrete correct answer, thus we included in this category all the answers that were correctly discussed.
In Figure 4 we show the results obtained by the students in the four previously mentioned institutions, ordered by the amount of low, medium and high level answers. As we can see, students perform worse when using the virtual visit application than just after the initial talk. That is a very interesting and intriguing fact, since the initial talk was performed always before the virtual visit and the contents were the same. Even if the virtual visit had no effect at all, results should remain the same. The only difference between both activities is the format and methodology of the activity, thus it is there where we should look for the origin of this paradoxical effect.

To analyze the reasons why students' performance decreases when using the application, we developed a second pilot experience in another education center, namely CEIP3, with students aged around 12. In this new experience, we divided students into two groups of 26 and 28 students,

For students in the first group we followed the protocol described previously, aiming at comparing the new results with the former ones. That is the reason why results obtained from this group are directly compared to those from figure 4 . For the second group, a similar scenario took place, but including a reward if they finished Practice 2 correctly. Results from this new experience, with and without rewards, are shown in Figure 5 .

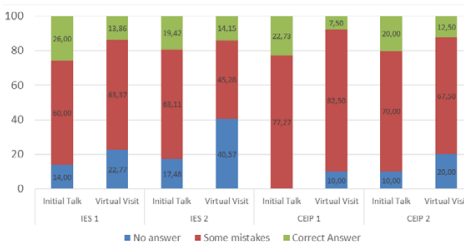

Fig. 4: Percentage of students that answered to the exercises right after the Initial Talk (Practice 1) and during the virtual visit (Practice 2) for the 4 education organizations

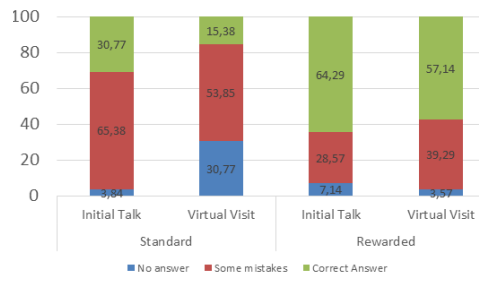

Fig. 5. Percentage of different answers from students when they are included in a group with our standard procedure and when we included a reward depending on their answers

In that figure, we can see that no rewarded students obtained similar results than the previously analyzed ones. These results are reasonable, since the only difference among them is the institution they belong to: students' age and protocol were the 
same. Again, those students performed worse while visiting the virtual site. Moreover, the number of students that did not give any answer at all increased significantly. On the other hand, students that answered correctly after the initial talk doubled those who did it after the virtual visit.

With respect to the kind of used rewards, they were related to allowing students to use the virtual visit as a ludic tool or to use any other game from their personal computers. This reward was directly related to their performance and students only had access to it right after they got a good evaluation by the teachers. Depending on their performance, teachers decided what kind of benefits students would get, being those proportional to students' performance. The performance of the group that worked under those rewarding conditions did not suffer the strong decreasing the other group did, as we can see in figure 5 . For example, we found out, for the first time, that the amount of empty answers decreased. Although the number of correct answers also decreased, this reduction is smaller, and highly compensated by the higher amount of students that were included in the intermediate category.

Finally, let us analyze the increasing in their knowledge due to the use of the virtual visit by measuring the correct answers from e-valUAM test, i.e. the last phase of the experience described in figure 3 . Thus, to measure the influence of the virtual visit in the learning process, we extended the experience to different scenarios, where the questionnaire was answered before and after using the virtual visit tool. We found an average score of 7.05 in the e-valUAM questionnaire from students who used the virtual visit before answering, and an average score of 6.51 from groups who answered the questionnaire before using the application. In this preliminary result we find an increasing in students' knowledge right after using the application. It is worth noting that, in this case, students did not have the opportunity to use the application at the same time. This fact reveals again that the performance reduction only happens when students use the application at the same time, being demonstrated that the virtual visit application is a distraction, most likely due to the high motivation of students to use it as a ludic activity.

\section{CONCLUSIONS}

We have presented a virtual visit of an Archeological site in Egypt as well as a methodology to use it as an e-learning tool. The methodology showed that the expectation and use of the application can affect students' performance when developing other activities that can be perceived as being less interesting. To avoid this effect, we propose to use the virtual visit in an independent session in order to avoid any possible distraction, or to apply gamification mechanics such as rewards.

We found in this study that the use of the virtual visit is a very exciting activity for the students, which make them forget the initial learning goals of the application. That result was obtained by measuring students' different performance between "practice 1" (right after the initial talk) and "practice 2" (being done in the computer laboratory at the same time than the virtual visit). In general, answers from the first exercise were longer and included a higher amount of significant information. This is a very important result since it demonstrates that the interest of the students in the virtual visit can be an obstacle for their performance in other tasks.

As future work, we plan to analyze the texts obtained in both Practice 1 and Practice 2 by emotion detection procedures [13] in order to measure students' motivation in both scenarios.

\section{ACKNOWLEDGEMENT}

This work has been partially supported by the projects TIN2013-44586-R, TIN2014-52129-R, TIN2014-56494-C4-4-P, FCT-14-8677 and S2013/ICE2715. GMS appreciates the support of the Spanish "Ramón y Cajal" program. The authors also wish to express their gratitude to the students who have participated in this experience during the 2015-2016 course.

\section{REFERENCES}

[1] Wu J. P., Tsai R. J., Chen C. C. and Wu Y. C (2006), An integrative model to predict the continuance use of electronic learning systems: hints for teaching. International Journal on E-learning, vol. 5(2), pp. 287-302.

[2] Molins-Ruano P., Borrego-Gallardo F., Sevilla C., Jurado F., Rodriguez P., Sacha G. M. (2014). Constructing Quality Test with e-valUAM. IEEE Xplore Proceedings of Computers in Education (SIIE), International Symposium on. pp 195-200.

[3] McGonigal J. (2011) Reality is broken: Why games make us better and how they can change the world. New York, NY: Penguin.

[4] Zichermann G. and Linder J. (2001) Game-based marketing: Inspire customer loyalty through rewards, challenges and contests. Hoboken, NJ: Wiley.

[5] Mozelius P., Collin J. and Olsson M., (2015) Visualisation and gamification of e-Learning - Attitudes among course participants. Proceedings of International Conference on e-Learning, Nassau.

[6] Serioa A. D., Ibáñez M. B. and Kloos C. D. (2013) Impact of an augmented reality system on students' motivation for a visual art course. Computers \& Education, vol. 68, pp. 586-596.

[7] Attali Y. and Arieli-Attali M., (2015) Gamification in assessment: Do points affect test performance?. Computers \& Education, vol 83, pp. 57 63.

[8] Shaffer D. W., (2006) How computer games help children learn. New York NY; Palgrave Macmillan.

[9] Jackson G. T., Dempsey K. B. and McNamara D. S., Game-based practice in a reading strategy tutoring system: Showdown in iSTARTME. (2012) In H. Reinders (Ed.), Digital games in language learning and teaching, pp. 115-138, Basinstoke, England: Palgrave, McMillan.

[10] Molinero-Polo M. A., Hernández C., Méndez-Rodríguez D. M., Naranjo T., Díaz Y., Pérez-Ruiz S., Acebo A., Molins-Ruano P., Jurado F., Rodriguez P., Atrio S. and Sacha G. M. (2016) Bringing Egyptology to the Classroom: Virtual Reconstruction of the TT 209 Site. IEEE Xplore Proceedings of the International Conference on Remote Engineering and Virtual Instrumentation (REV'16) 306-310

[11] Molins-ruano P., González-sacristán C., Diez F., Rodriguez P. and Sacha G. M. (2015) An Adaptive Model for Computer-Assisted Assessment in Programming Skills. International journal of Engineering Education, vol. 31 , pp. 764-770.

[12] Molinero-Polo M. A., Pérez-Ruiz S., Acebo A. and Sacha G.M. (2016) Análisis del potencial motivador y docente del uso de las nuevas tecnologías en el aula. Interacción entre el aumento de la motivación y el aprendizaje. Invited article in "Cambios metodológicos: nuevas formas en la enseñanza" de la revista OGE (Organización y Gestión Educativa) from fórum europeo de administradores de la educación. Accepted 2016.

[13] Molins-Ruano P., Sevilla C., Santini S., Haya P. A., Rodríguez P. and Sacha G. M. (2014) Designing videogames to improve students' motivation," Computers in Human Behavior, vol. 31 pp. 571-579. 